\title{
New enzyme immunoassay for detecting cryptococcal antigen
}

\author{
F R Knight
}

\begin{abstract}
Results obtained with a recently introduced enzyme immunoassay system (EIA) for the detection of cryptococcal antigen (Meridian Diagnostics Inc) were compared with those obtained by a latex agglutination (LA) method (ImmunoMycologics, Norman, Oklahoma, USA). Fifty four samples were examined. There was $92 \%$ agreement between the two methods. One false positive result was obtained with LA, and one sample was inevaluable. The EIA was rapid and simple to perform. There was some evidence that it gave fewer false positive reactions and improved the diagnosis of genuine early cases.
\end{abstract}

Cryptococcus neoformans is an increasingly important cause of infection, particularly in the immunosuppressed. Since 1984 cases dealt with by the Mycological Reference Laboratory (MRL) have increased more than fourfold (from 14 to 64 a year). Currently, $78 \%$ of these patients are known to be human immunodeficiency virus (HIV) positive (1991 figures). Cryptococcosis is now the fourth most common cause of opportunistic infection in patients with AIDS. ${ }^{1}$ In the United Kingdom it affects $4 \%$ of that patient group.

The Premier system is an enzyme immunoassay (EIA) procedure in which polyclonal antibody to $C$ neoformans has been coated on to plastic microwells. For convenience, these are constructed so that strips containing the requisite number of microwells can be detached from the plate. The detection system is based on a monoclonal antibodyperoxidase conjugate. If cryptococcal antigen is present in the sample, it forms a complex with enzyme conjugate and adsorbed antibody. After washing to remove unbound conjugate, a substrate solution is added. The amount of colour developed is related to the quantity of cryptococcal antigen present.

\section{Method}

Mycological Reference Laboratory, Central Public Health Laboratory, 61 Colindale Avenue, London NW9 5HT

F R Knight

Correspondence to: F R Knight

Accepted for publication 31 January 1992
A total of 54 samples (39 sera, 14 spinal fluids, and one urine) were submitted to the MRL for testing. The samples were from 33 patients, 15 of whom were known to be HIV positive. Specimens were from confirmed cases of cryptococcosis, patients in whom the diagnosis was not subsequently confirmed, four patients with other mycoses (histoplasmosis, aspergillosis, candidosis and farmers' lung) and a pool of sera from healthy subjects. Samples had been stored at $-30^{\circ} \mathrm{C}$ after LA testing, before testing by EIA.

Samples were examined according to the manufacturer's instructions for both LA and EIA. For EIA, samples known to be HIV positive were also heated to $56^{\circ} \mathrm{C}$ for 30 minutes before testing. The LA test includes this heating stage as part of the detacher enzyme pretreatment of samples. EIA results were compared with those obtained with LA. To conserve reagent, only selected samples were titrated to end point with the EIA. These were determined at sample dilutions of 1 in 2 to 1 in 1250 , as recommended by the manufacturers.

\section{Results}

Of the samples examined and compared, 28 were positive with LA (20 sera, seven cerebrospinal fluid, and one urine), and 24 $(86 \%)$ of these were initially positive with the EIA. A further two samples were positive on further testing at a lower dilution (1 in 1), giving a figure for agreement of $92 \%$. Two samples remained negative.

One of the negative samples had an LA titre of $1 / 5$. No clinical details were provided, and no further samples were available for testing. The other had an original LA titre of $1 / 1000$, but there was insufficient sample for repeat testing. However, a later sample from the same patient was negative on testing with LA. Excluding this result gives an agreement of $96 \%$ between the two tests.

Samples giving positive reactions by EIA were not quantified in all cases. Of those that were, comparisons between EIA and LA results are shown in table 1 .

In five samples the EIA titres were lower than the LA titres and in 11 samples they were

Table 1 Comparison of results with ELA and LA

\begin{tabular}{llll}
\hline Sample reference & Specimen & ELA titre & LA titre \\
\hline MB 01.03.89 & Serum & $1 / 1877$ & $1 / 200$ \\
MB 02.11.89 & Serum & $1 / 25$ & $1 / 200$ \\
MB 22.01.91 & Serum & $1 / 4$ & $1 / 200$ \\
MB 25.04.91 & Serum & $1 / 4$ & $1 / 20$ \\
TB 21.09.89 & Serum & $1 / 6315$ & $1 / 2000$ \\
TB 07.09 .89 & Urine & $1 / 17$ & $1 / 50$ \\
AA 11.03 .91 & Serum & $1 / 11$ & $1 / 5$ \\
AA 26.03.91 & Serum & $1 / 17$ & $1 / 5$ \\
MC 08.08 .89 & CSF & $1 / 2453$ & $1 / 100$ \\
MC 23.08.89 & CSF & $1 / 1325$ & $1 / 50$ \\
MS 13.05 .91 & Serum & $1 / 610$ & $1 / 200$ \\
M2 13.05 .91 & Serum & $1 / 96$ & $1 / 5$ \\
TT 17.05 .91 & Serum & $1 / 2913$ & $1 / 200$ \\
LT 17.05 .91 & Serum & $1 / 1765$ & $1 / 200$ \\
LT 16.05 .91 & CSF & $1 / 10247$ & $1 / 400$ \\
\hline
\end{tabular}


Table 2 Comparison of ELA absorbance readings at OD450 for sera not heated/heated to $56^{\circ} \mathrm{C}$

\begin{tabular}{lll}
\hline Sample reference & $\begin{array}{l}\text { Absorbance reading } \\
\text { (NOT heated) }\end{array}$ & $\begin{array}{l}\text { Absorbance reading } \\
\text { (heated) }\end{array}$ \\
\hline MB 01.03.89 & 1.697 & 1.742 \\
MB 02.11.89 & 1.773 & 1.920 \\
MB 22.01.91 & 0.512 & 0.663 \\
\hline
\end{tabular}

higher. However, direct comparison of titres was not possible because samples were stored between LA and EIA testing. Nevertheless, it is interesting to compare the three sera from case MB for which both LA and EIA titres are available (table 1). The three sera were received at intervals of eight months and 27 months from the initial specimen. If storage does affect the results, it does not have the same effect on all samples.

There were 26 negative samples by both EIA and LA tests. Included in this group were the four samples from patients with other mycoses and the pool of sera from healthy subjects. In no instance was a sample positive with EIA and negative with LA, though again it would be unfair to draw any conclusions from this without examining samples prospectively with both test systems. Recently, Gade et $a^{2}$ compared the Premier EIA with the Calas LA system and obtained three false positive results with the EIA from 475 specimens tested. Five other samples were originally EIA positive/LA negative, and became LA positive on testing of subsequent samples.

In the current study all sera from HIV positive patients were heat inactivated at $56^{\circ} \mathrm{C}$ for 30 minutes before testing. Three sera tested with and without heating showed that absorbance readings were only marginally increased on heating (table 2). This would make little difference in practice as all samples from an individual patient would be similarly treated.

\section{Discussion}

Of particular interest are the discrepancies between results with the two procedures. In our experience $0.8 \%$ (six out of 880 in 1991) of positive LA tests for cryptococcosis have titres of $1 / 5$ or lower, which are negative on testing a subsequent sample. In practice such patients are rarely a problem. When a first sample gives a low LA titre, a repeat sample is requested. If this sample is still positive then an early infection is suspected. Patients with cryptococcosis usually present with much higher titres.

Two samples were positive by LA but negative by EIA. One of these is inevaluable but may be a false positive. The other discrepant sample had an LA titre of $1 / 1000$ but a negative EIA result. However, a subsequent sample from this patient was negative by LA. In our experience this is a very unusual finding. In all previous cases where LA titres had exceeded $1 / 5$, overt cryptococcosis had been confirmed. It is concluded that the initial LA result was false positive, perhaps because the detacher enzyme used to treat the sample was deficient through poor storage, or because the quantity added was insufficient.

Other samples gave different results with the two test systems. One (AA) gave identical LA titres $(1 / 5)$ on testing of consecutive samples and titres of $1 / 11$ and $1 / 17$, respectively, for EIA. This patient was HIV positive, and a subsequent sample examined five months later, by which time cryptococcal meningitis had been diagnosed, had an LA titre of $1 / 200$.

In another case (M2) titres with LA and EIA tests were $1 / 5$ and 1/96, respectively. As both patients appeared to have true infections, results with EIA may have correlated better with the clinical status than those obtained with LA.

One case (PO) with culturally confirmed cryptococcosis was repeatedly negative with both LA and EIA. This is the first patient we have seen with a true infection whose antigen titres were consistently negative (case report to be published). In this instance it may be that the level of antigen present is below the detection threshold for both tests.

The EIA test was easy to perform, a screening test taking no longer than the LA test. Titration of first time positive results would take longer, but in practice most samples for titration are from known positive results in whom the screening test would be omitted. Further studies are needed to show if EIA titres are clinically useful in following disease progression/remission.

On the basis of existing prices, the cost per test for both systems is comparable (about £2).

The simplicity, rapidity and reliability of this new EIA test makes it a useful laboratory aid for the diagnosis of a life threatening and increasingly common opportunistic mycosis.

We are grateful to Launch Diagnostics Ltd, for providing the PREMIER EIA kits used in this study.

1 Patterson TF Andriole VT Current concepts in cryptococcosis. Eur 7 Clin Microbiol Infect Dis 1989;8:457-65. Gade W, Hinnefeld SW, Babcock LS, et al. Comparison of the Premier cryptococcal antigen enzyme immunoassay and the latex agglutination assay for detection of cryptococcal antigens. $\mathcal{f}$ Clin Microbiol 1991;29:1616-19. 